Commun. Fac. Sci. Univ. Ank. Ser. A1 Math. Stat.

Volume 69, Number 1, Pages 276-306 (2020)

DOI: $10.31801 /$ cfsuasmas. 542499

ISSN 1303-5991 E-ISSN 2618-6470

http://communications.science.ankara.edu.tr/index.php?series=A1

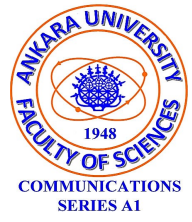

\title{
CONFIDENCE REGIONS FOR BIVARIATE PROBABILITY DENSITY FUNCTIONS USING POLYGONAL AREAS
}

\author{
ORHAN KESEMEN, EDA ÖZKUL, AND ÜLKÜ ÜNSAL
}

\begin{abstract}
In this study, a polygonal approach is suggested to generalize the notion of the confidence region of the univariate probability density function for the bivariate probability density function. The equal density approach is used to demonstrate that confidence regions can be polygonal shapes. The bisection method is the preferred method in finding the equal density value that reveals the desired confidence coefficient. Confidence regions estimate not only bivariate unimodal probability functions but also bivariate multimodal probability functions. An approach is enhanced to estimate these confidence regions for probability density functions which are defined as rectangular, polygonal and infinite expanse areas. In order to show the applicable of the proposed method, four different examples are analyzed. The results show that the confidence region is found no matter how complex the distribution function. In addition, the proposed method gives more efficient results for multimodal probability density functions.
\end{abstract}

\section{INTRODUCTION}

In statistics, a confidence interval is an estimation of a parameter which represents the population within an acceptable range. Confidence interval was firstly identified, and its validity was proven by Neyman [1]. Tate and Klett [2] determined the optimal confidence interval and they estimated the optimal confidence interval for a normal distribution. Then, Dunn 3, 4 presented several procedures for determining the rectangular confidence regions. Chew 5 compiled the formulas for confidence, prediction, and tolerance regions for the multivariate normal distribution for the various cases of known and unknown mean vector and covariance matrix. Sidak [6] proved the validity of the rectangular confidence regions for the means of multivariate normal distributions given by Dunn [3, 4]. Hu and Yang [7] proposed a distribution-free approach, based on a few basic geometrical principles, to determine the confidence region for two or more variables. Also, they analyzed

Received by the editors: March 20, 2019; Accepted: October 03, 2019.

2010 Mathematics Subject Classification. Primary 62F25; Secondary 62 E15.

Key words and phrases. Bivariate confidence region, bivariate probability density functions, multimodal confidence region, polygonal confidence region.

(C) 2020 Ankara University Communications Faculty of Sciences University of Ankara-Series A1 Mathematics and Statistics 
some biological data sets to demonstrate the use of the proposed method for genomics. Mammen and Polonik [8] constructed a confidence region for a density level set using kernel density estimators. Martin [9] described an approach, based on random sets, to construct exact confidence regions that attain the nominal coverage probability. Rambaud et al. [10] used the confidence regions to determine the characteristics for diagnosis of pneumonia in children younger than 5 years. Harrar and $\mathrm{Xu}$ [11] developed methods to construct confidence regions for level differences in the multi-dimensional cases and they applied it to the profile analysis.

This paper proposes the confidence regions for bivariate probability density functions using polygonal areas. The aim of this study is to estimate a more accurate confidence region by utilizing the equal density approach.

\section{Confidence Interval}

In a probability density function, infinite confidence interval might be defined that gives same confidence coefficient. Three different approaches are improved for choosing the most convenience in these intervals. These approaches are

a) The equally tailed confidence interval,

b) The shortest confidence interval,

c) The equal density confidence interval.

These methods show the same limits in symmetric distribution, such as normal distribution. However, the shortest confidence interval and the equal density confidence interval estimate the same limits while the equally tailed confidence interval estimates different limits in asymmetric distributions [8, 12, 13, 14]

Suppose $\mathbf{X}=\left\{X_{1}, X_{2}, \ldots, X_{n}\right\}$ are random samples that are taken from an i.i.d. This distribution's probability density function is $f(x ; \theta, \ldots)$ and the parameter $\theta$ is defined $\theta \in \mathbb{R}$. The values $\mathbf{x}=\left\{x_{1}, x_{2}, \ldots, x_{n}\right\}$ are the observed values of $\mathbf{X}$. Interval estimation of $\theta$ for these observed values are executed by means of the two bound values $L$ and $U$. These two limit values must satisfy the condition $L \leqslant U$ for all $x$ values. As a result, interval $[L, U]$ should involve the parameter $\theta$ with a certain probability $(1-\alpha)$. This probability value is called a confidence coefficient. This confidence coefficient constitutes the confidence interval when it is used in a format such as (1).

$$
\operatorname{Pr}(L \leqslant \theta \leqslant U)=1-\alpha
$$

Here, the value $\alpha$ is the significance coefficient which is determined by the researcher and it ranges from $(0,1)$.

2.1. The Equally Tailed Confidence Interval. The equally tailed confidence interval is used commonly in the literature. Statistic $\theta^{*} \sim \Phi(x)$, which is obtained from a sample, is an estimation of the parameter $\theta$ of the discussed population. If the following condition is satisfied, this interval is called the equally tailed confidence interval. 


$$
\operatorname{Pr}[L \leqslant \theta \leqslant U \mid \operatorname{Pr}(\theta \geqslant U)]=1-\alpha
$$

According to this condition, the probabilities of parameter $\theta$, being less than the lower bound and being greater than the upper bound, are equal. Therefore, in this interval, the right and the left side of the confidence region are equal to each other.

The parameter $\theta$ of the population with the $(1-\alpha)$ confidence coefficient can be calculated with the inverse distribution function below.

$$
\operatorname{Pr}\left[\Phi^{-1}\left(\alpha / 2 ; \theta^{*}, \ldots\right) \leqslant \theta \leqslant \Phi^{-1}\left(1-\alpha / 2 ; \theta^{*}, \ldots\right)\right]=1-\alpha
$$

2.2. The Shortest Confidence Interval. The shortest confidence interval is defined as (4).

$$
\operatorname{Pr}[L \leqslant \theta \leqslant U \mid \min (U-L)]=1-\alpha
$$

There are infinite confidence limits with the same confidence level. In this method, confidence limits which have the minimum confidence width $(U-L)$ is preferred. Although the shortest confidence interval gives a more precise estimation, it is not preferred due to difficulty of its calculation.

2.3. The Equal Density Confidence Interval. The equal density method presents a different approach to the shortest confidence method. The basis of this approach is the conditional equality which is given as follows.

$$
\operatorname{Pr}[L \leqslant \theta \leqslant U \mid \phi(L ; \theta, \ldots)=\phi(U ; \theta, \ldots)]=1-\alpha
$$

According to (5), the probability density values are equal in the shortest confidence limits with the $(1-\alpha)$ confidence coefficient.

This approach is novel because it determines a cutting level in the y axis rather than research shortest confidence interval in the $\mathrm{x}$ axis, in order to calculate the shortest confidence interval. According to the cutting level, the confidence limits are determined as the roots of the following equation.

$$
\phi(x ; \theta, \ldots)-\zeta=0
$$

This method is superior because it determines the confidence interval within a multimodal probability density function [14].

\section{Confidence Region in Polygonal Area}

The univariate confidence region is defined as a region that is restricted by the two bounds, $(L, U)$, of the probability density function. These bounds can be determined by employing the afore-mentioned methods. However, the confidence region for bivariate probability density function cannot be estimated by using the equallytailed confidence interval. In this case, the confidence region with the smallest area can be searched. Although there are many regions with the same volume, the 
confidence region with the smallest area can be found by utilizing two objective optimization techniques [13. Since there is no relationship between two objective functions in multi objective optimization problems, several solutions with the same $(1-\alpha)$ confidence coefficient are selected to solve the problem, and the region with the smallest area is considered the confidence region. Although the selected region is likely the desired region, it is possible for deviations to exist. The aim of this study is to estimate a more accurate confidence region by utilizing the equal density approach.

3.1. Determining the Search Area for the Confidence Region. It is often impossible to determine a function, which defines the bounds of the region, that estimates a confidence region for bivariate arbitrary probability density functions. To solve this problem, this study considers the confidence region to be polygonal region. A polygonal region is a closed region which is formed by lines with combining clockwise or counterclockwise set points. The determination of this region on an infinite plane is often not possible due to the required computation time and memory usage. To solve this problem, an approximate search area is determined according to the probability density function within various situations, and thus the confidence region can be estimated with greater accuracy in this region. The determination of the search area for some probability density functions is given below.

3.1.1. The Search Area for the Rectangular Definition Region. In Figure 1(a), the probability density function of the discussed statistics is defined in a polygonal (rectangular) region $\left(\Phi(x, y):[a, b] \times[c, d] \rightarrow \mathbb{R}^{2}\right)$. Its search area $\left(\Omega=\left\{P_{i}=\left(x_{i}, y_{i}\right), i=1,2, \ldots, 4\right\}\right)$ is described in Figure 1 (b).

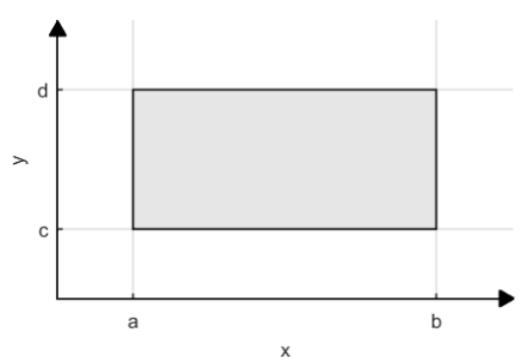

(a)

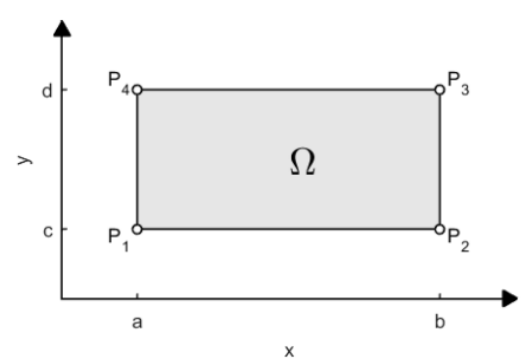

(b)

Figure 1. Determination of the search area of the rectangular definition region, (a) Rectangular definition region, (b) Search area. 
3.1.2. The Search Area for the Arbitrary Definition Region. In Figure 2(a), the probability density function of the discussed statistics is defined in an arbitrarily restricted area. This area can be taken from digital images, maps, satellite images etc. There are many applications of bivariate density functions that are in a region restricted by polygonal area. These applications include the analysis of pollution or crime rate in a city, of the earthquake risk distribution and frequency in a country, of the density of a certain tree species in a forest 15, of the intensity of the dispersion of harmful insects in a field, of the traffic density in a particular area and of the location and transmission of an epidemic illness in a country. In addition, there could be multiple piecewise density functions of the examined area in the polygonal region. In this regard, the applications of bivariate density functions extend to many physically and politically divided cities (eg. Belfast, Beirut, Jerusalem, Mostar and Nicosia). That said, the data in the politically divided cities changes significantly over time. In this case, it may not be possible to evaluate an entire city in the same way that a region is evaluated. Each example may need statistical analysis based on a probability density function that is defined within a region with arbitrarily determined limits. A polygonal structure should be used to define an arbitrary region. Manual or automatic identification can determine the nodes of the polygonal area. In automatic identification, the examined region must be converted to a digital image. The nodes of the objects in the digital images are automatically determined by using the dominant point detection algorithm [16. In this case, the search area, $\Omega=\left\{P_{i}=\left(x_{i}, y_{i}\right), i=1,2, \ldots, N_{\Omega}\right\}$, is defined as a polygonal region consisting of $N$ nodes.

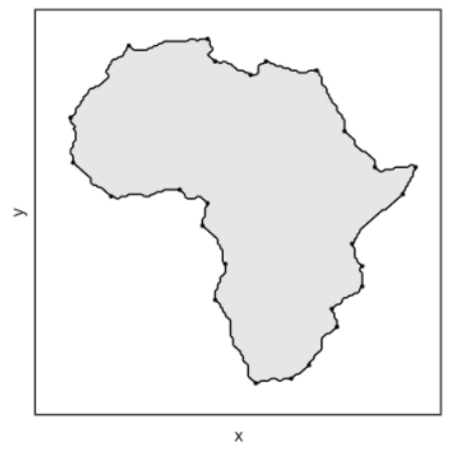

(a)

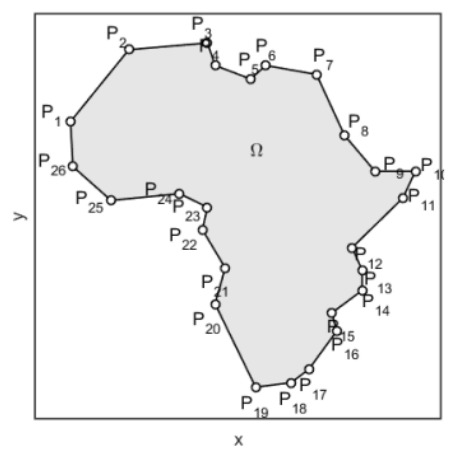

(b)

Figure 2. Determination of the search area of the polygonal definition region (a) Arbitrary definition region (African content) (b) Converting the arbitrary region to the polygonal search area via dominant points. 


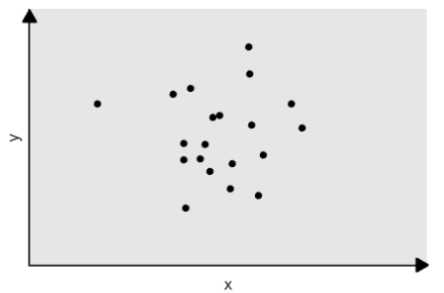

(a)

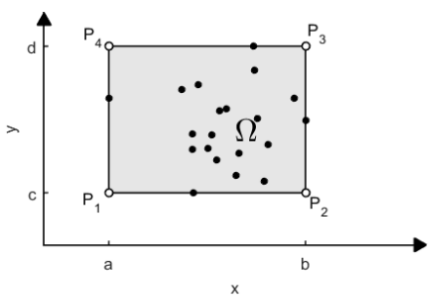

(c)

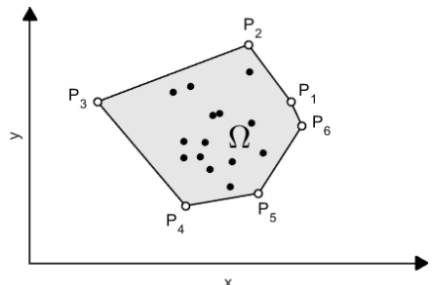

(b)

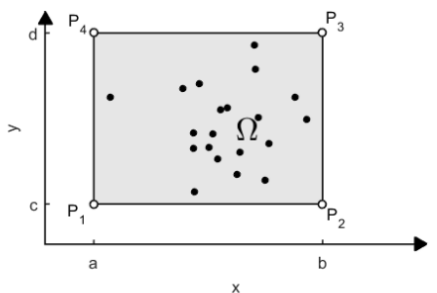

(d)

FiguRE 3. Determination of the search area via the points which are randomly generated from the distribution function, (a) Randomly generated points in an infinite definition region (b) Definition of the polygonal search area consisting of the random points (c) Determination of the rectangular search area on the boundaries of the random points (d) Determination of the expanded search area.

3.1.3. The Search Area for the Infinite Definition Region. The probability density functions are often defined in an infinite or semi-infinite space. In this study, the search area is determined as a finite region which has a higher confidence coefficient instead of an infinite region due to the difficulty of searching within an infinite region. In this regard, the proposed automatic solution requires generating a sufficiently random number(point) from the probability density function in this region (Figure 3(a)). This study proposes two approaches to determine the search area via these random points [17.

The first approach defines a polygonal search area $\Omega=\left\{P_{i}=\left(x_{i}, y_{i}\right), i=1,2, \ldots, N_{\Omega}\right\}$ (Figure 3(b)) in which each point in this convex polygon surrounds random points called nodes The second approach determines a rectangular search area (Figure 3(c)) consisting of bounds of randomly generated numbers $(\Omega:[\min (X), \max (X)] \times$ $[\min (Y), \max (Y)])$. However, in order to find the confidence region within the desired level for each of these two approaches, generated numbers must be numerous or selected search area must be expanded (Figure 3(c)). 
3.2. Triangulation of the Search Area. In this study, a polygonal approach is proposed to find the confidence region within the search area. The equal density approach can be only used to find the coordinates of the nodes of the polygonal confidence region because there is no tail definition for equally area or equally volume in the two-variable expression. In addition, the term of the shortest region converts to the two objective optimization problem. In this optimization problem, the reliability of computation is weak because there is no relationship between objective functions. As the equal density approach creates polygonal closed curves similar to contour curves, it becomes simpler to apply as compared to the other approaches. In order to more quickly reach the solution in an infinite space, the search area is divided into small triangles and then the nodes of the polygonal confidence region are sought in the edges of these triangles.

If the search area has a polygonal structure, grid points that are created around the polygon equidistantly in rectangular area are selected via the minimum and the maximum coordinates of the polygon nodes. These points are then recorded in a list (Figure 4(a)). Grid points that fall out of the polygon are removed from the list. The remaining grid points and nodes within the polygon are recorded on the same list, and obtained point set are divided into small triangles by using the Delaunay triangulation algorithm 18 (Figure 4(b)). If the polygonal confidence region needs to be more sensitively bound, then the region should be gridded more closely and divided into more small triangles (Figure 5 .

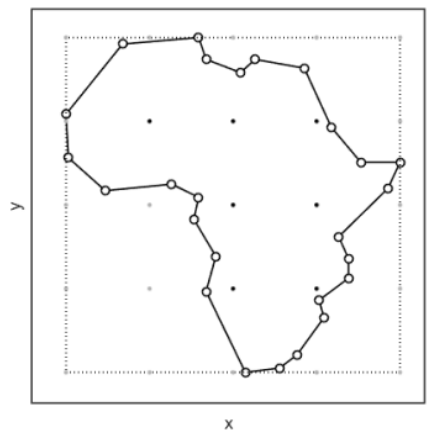

(a)

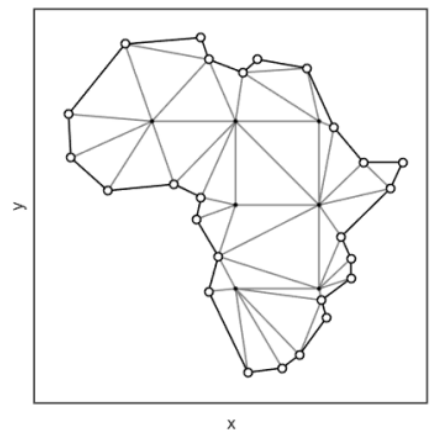

(b)

Figure 4. Dividing the polygonal area into small triangles, (a) Choosing the grid points with the size $5 \times 5$ grid; (b) Triangulation of the polygonal area.

When the number of grids increases, the sensitivity of confidence region also increases. Computation time and memory usage also increase. Therefore, the choice of the number of grids belongs to the researcher. However, it can be easily applied if the search area has a rectangular shape. 


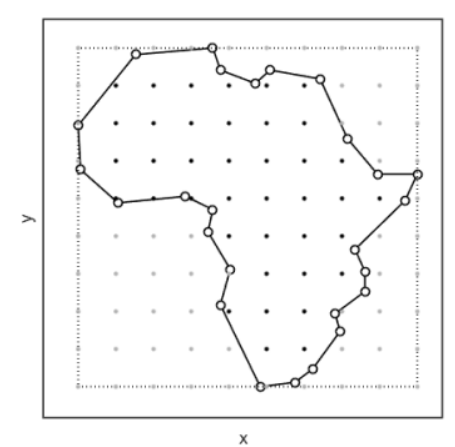

(a)

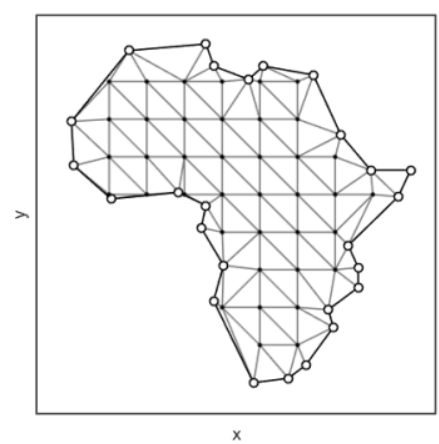

(b)

Figure 5. Dividing the polygonal area into small triangles, (a) Choosing the grid points with the size $10 \times 10$ grid; (b) Triangulation of the polygonal area.

There are many studies in the literature about the optimal grid size for calculating numerical integration [19, 20, 21, 22,. Since this study is based on statistical distributions, it is necessary to investigate whether the error is significant or not. Selected statistical distributions can be used in many polygonal areas with different parameters. Also, distributions in the real life may be mixed or truncated distributions. In that case, it must be multiplied by a constant c, for the summation of the distribution value to be 1 . Therefore, the constant $\mathrm{c}$ depends on the number of the selected grids. The best approach is to select the number the grid size according to the researcher's purpose. Nevertheless, the following equation can be given as an appropriate approach to determine the number of grids in each case.

$$
I=\arg \min _{i=1,2, \ldots}\left|\frac{\Phi_{i}-\Phi_{i-1}}{\Phi_{i}}-\epsilon\right|
$$

This equation gives the number of grids of two regions. $\Phi_{i}$ is the total volume of the two grids and $\epsilon$ is the tolerance value.

3.3. Computing the Probability in the Polygonal Area. The volume between the surface of the bivariate probability density function, defined in polygonal area and $\mathrm{x}-\mathrm{y}$ plane, gives the sum of the probability value in the polygonal area. Hence, the probability value is calculated for each triangle within the triangulated polygonal region in order to estimate this volume. Analytical and numerical approaches for the calculation of the probability in the polygonal region are improved by Kesemen et. al 15 .

3.4. Determining the Polygonal Area for the Cutting Level. In this study, the equal density approach is used to determine the confidence region from the 
probability density function which is defined in the polygonal area. To explain the equal density approach, the model, shown in Figure 6, is chosen as an example. Figure 6(a) shows the values of two variable probability density functions in a polygonal area according to color changes. These color changes are shown in the surface form in Figure 6(b).

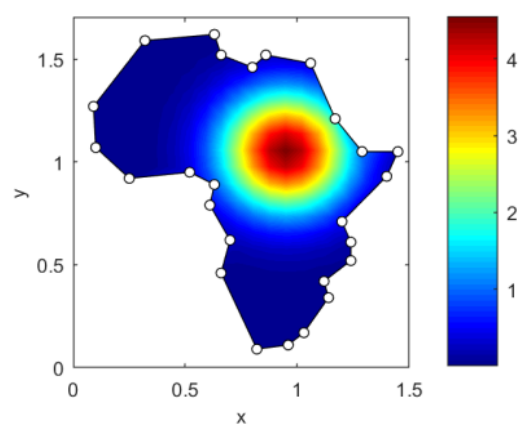

(a)

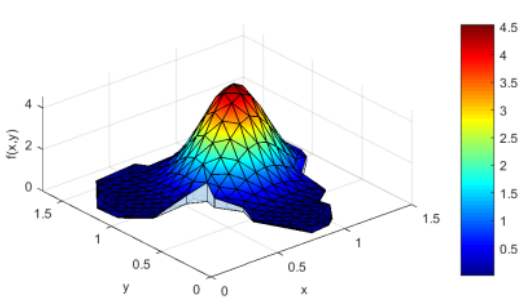

(b)

Figure 6. Normal distribution which is limited by the African continent, (a) Contour format; (b) Surface format.

Figure 7 (a) shows the intersection of a plane at $\zeta$ level. It is selected parallel to the $\mathrm{x}-\mathrm{y}$ plane of the model given in Figure 6(b), with the probability density function placed in a polygonal area. The probability density function, which is intersected by $\zeta$ plane, returns to an area of zero under the plane. The area over the plane remains the same (Figure 7 (b)).

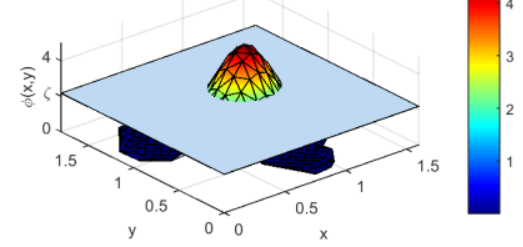

(a)

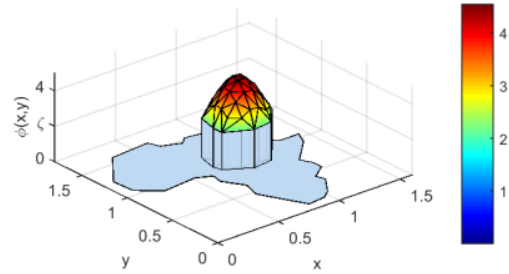

(b)

Figure 7. Cutting region, (a)Applying the cutting level to the probability density function; (b) Partial probability density function in the cutting region.

Each line segment between two nodes in the triangulated search area is labeled. Each labeled line segment and its neighbor nodes are recorded in a list. If the line 
segment is one of the edges of the polygon, it has only one neighbor node (Figure 8(a)). If not, it has two neighbor nodes (Figure 8(b)).

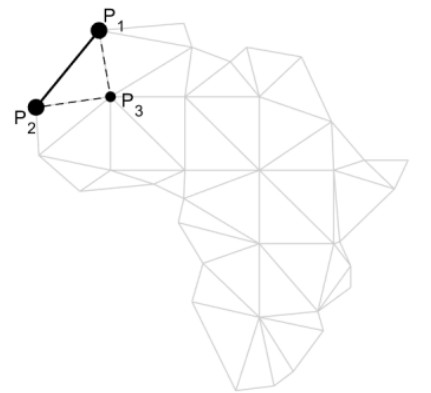

(a)

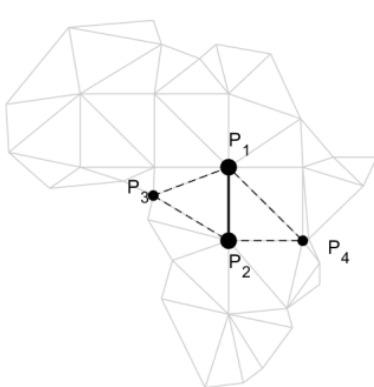

(b)

Figure 8. Labeling line segments, (a) Edge line segment and its neighbor nodes; (b) Inner line segment and its neighbor nodes.

The bold line segment in Figure 8 (a) is between $P_{1}$ and $P_{2}$. Since it is an edge of the polygon, it has only one neighbor node $\left(P_{3}\right)$. The bold line segment in Figure 8(b) is between $P_{1}$ and $P_{2}$. Since it is not an edge of the polygon, it has two neighbor nodes $\left(P_{3}\right.$ and $\left.P_{4}\right)$.

Two-dimensional linear interpolation is used for the determination of the cutting points of all lines cut by the $\zeta$, which is chosen by employing the equal density approach. According to interpolation, if the cutting point of each line is on the line segment, it is recorded as the inner polygon node (Figure 9(a), Figure 10(a), Figure 11(a)). These nodes $Q_{\zeta}:\left\{q_{i}=\left(x_{i}, y_{i}\right), i=1,2, \ldots, N_{Q}\right\}$ are listed by the line segment labels. The inner polygon nodes must be labeled in order to form a polygon. For this, in this study, the neighbor-tracking approach is used. According to this approach, labeling is performed by tracking the cutting nodes on the line segments (the dashed lines in Figure 11) in the neighbor nodes starting from an initial point (Figure 9(b), Figure 10(b), Figure 11(b)). If all cutting nodes are on the inner lines of the polygon, an inner polygon is formed by starting a random node and tracking the desired direction (Figure 9).

However, some cutting nodes fall on the line segment (Figure 10). Any edgecutting node is chosen as the initial point. Then, tracking is performed from the initial point to the inner cutting nodes. When an edge-cutting node is reached, the tracking process continues until it reaches the initial cutting point. Thus, the cutting polygon is determined. After tracking the second edge cutting node, the edge node with the highest density value is most preferred. In Figure 10(b), this node is shown in a square by label 20 .

If the cutting points cut the edges piece by piece (Figure 11(a)), tracking is performed from any edge cutting point to the inner nodes (Figure 11(a)). If an 


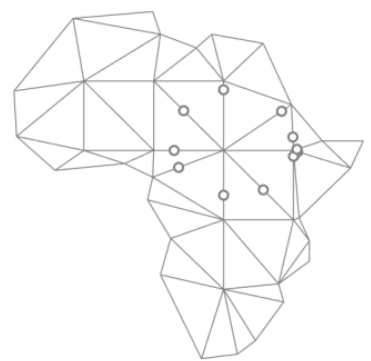

(a)

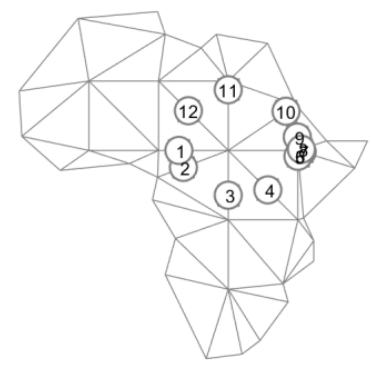

(b)

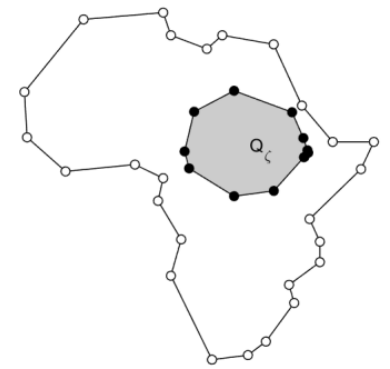

(c)

Figure 9. Inner polygon nodes according to selected cutting level, (a) Inner polygon nodes; (b) Sequentially labeled inner polygon nodes; (c) Inner polygon area.

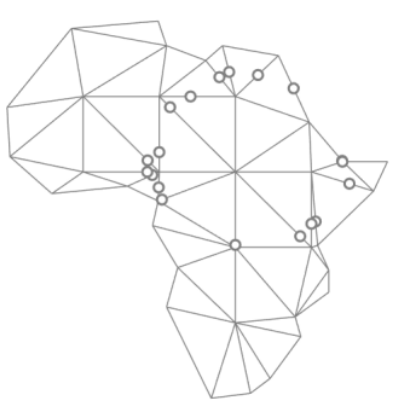

(a)

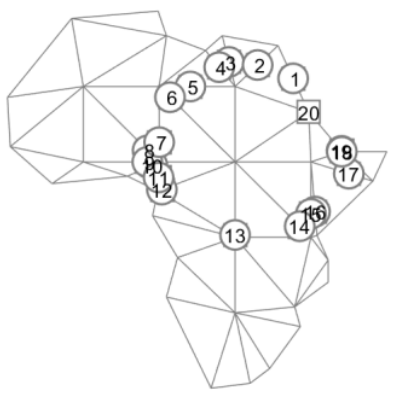

(b)

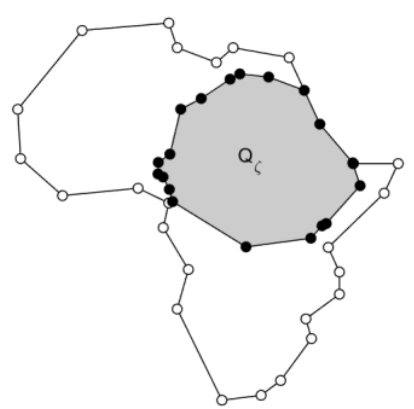

(c)

FiguRE 10. Inner polygon nodes according to selected cutting level, (a) Inner polygon nodes; (b) Sequentially labeled inner polygon nodes; (c) Inner polygon area.

edge node is reached in the tracking process, the tracking is continued on the edge node (Figure 11(a)) until another edge node is reached. If the reached node is not on the tracking list, tracking is performed on the other nodes (Figure 11(a)). This process is continued until the edge cutting node is on the tracking list (Figure 11).

Although the tracking process is finished, there are still unlabeled cutting nodes remaining. This shows that there is more than one confidence region. In this case, independent regions are determined by performing an independent tracking process to the unlabeled nodes.

3.5. Finding the Confidence Region within the Search Area. There is a relationship between the probability value $(P)$ of each level at the cutting region 


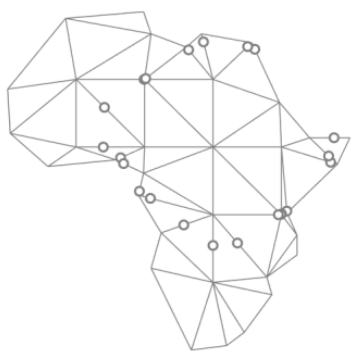

(a)

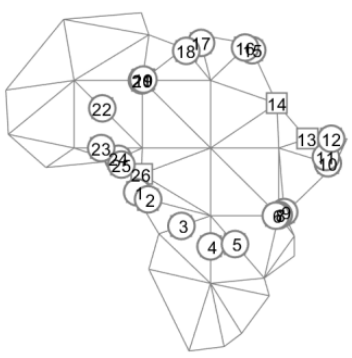

(b)

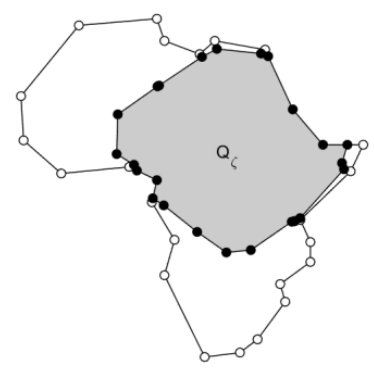

(c)

Figure 11. Inner polygon nodes according to selected cutting level, (a) Inner polygon nodes; (b) Sequentially labeled inner polygon nodes; (c) Inner polygon area.

and the cutting level $(\zeta)$. Based on this relationship, the probability value can be written as a function of $\zeta$ below.

$$
P(\zeta)=\operatorname{Pr}\left[(X, Y) \in Q_{\zeta} \mid \phi\left(x_{i}, y_{i}\right)=\zeta, i=1,2, \ldots, N_{Q}\right]
$$

In this approach, the bisection method is preferred in order to find the density level $(\zeta)$ which gives the desired probability value $(1-\alpha)$. According to the bisection method, two initial values, given below, are determined as the minimum and maximum value of the probability density value of all nodes.

$$
\begin{aligned}
\zeta_{a} & =\min \left\{\phi\left(x_{i}, y_{i}\right)\right\},\left(i=1,2, \ldots, N_{T}\right) \\
\zeta_{b} & =\max \left\{\phi\left(x_{i}, y_{i}\right)\right\},\left(i=1,2, \ldots, N_{T}\right)
\end{aligned}
$$

The probabilities of these levels are expected to be as follows.

$$
\begin{aligned}
& P\left(\zeta_{a}\right)=1 \\
& P\left(\zeta_{b}\right)=0
\end{aligned}
$$

This only occurs if the distribution function of the polygonal region is the uniform distribution. In this case, polygon points are collapsed inward or expanded outward until the desired probability value is obtained. Eventually, polygonal bounds of the region, which provide the desired confidence coefficient within a polygonal search area, are determined. 


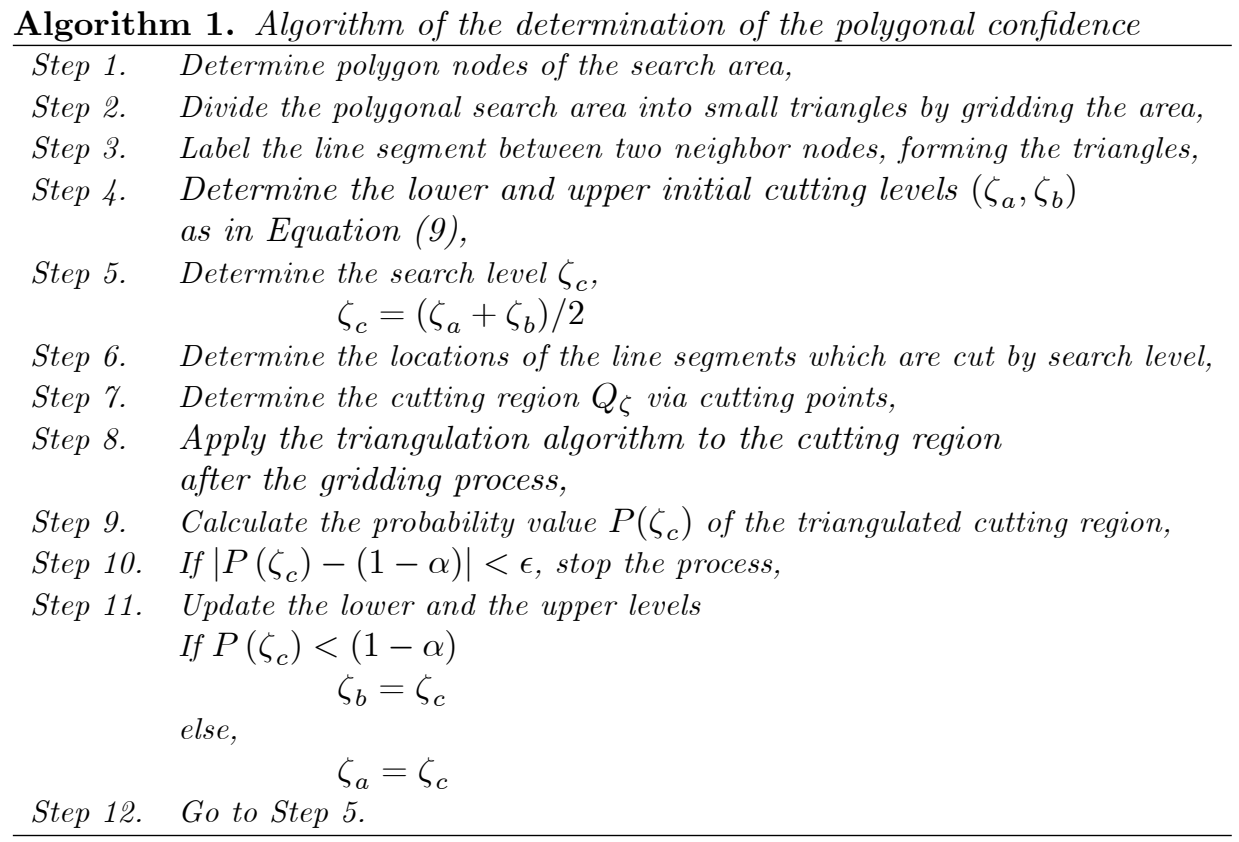

\section{Experimental Results}

In this study, three different examples are used to measure the performance of the proposed method. The first example aims to determine the confidence region for the standard normal distribution that is defined in an infinite space. In this example, the performance of the proposed method is shown by comparing the polygonal confidence regions. The second example searches the confidence region for the quadratic gamma distribution that is defined in a semi-infinite space. In the third example, the performance of the proposed method for multimodal probability density function is also analyzed. The fourth example examines the degree of influence by the northern Anatolian fault line of Sivas province.

For this study, a computer with an Intel ${ }^{\circledR}$ Core i7 $2.40 \mathrm{GHz}$ processor was used, and MATLAB ${ }^{\mathbb{R}}$ was used for the application. For all models, the contour that passes the through the middle of the initially selected minimum and maximum contours are determined in each iteration and then, the cumulative distribution value of its region is calculated. Until the obtained value converges the desired confidence level, the iterations are continued. The last found contour is assumed the desired contour.

Example 1. This example determines the confidence region with the confidence coefficients $\alpha=0.01,0.025,0.05,0.1$ for the bivariate standard normal distribution function defined in an infinite space. Its probability density function is given in Equation (11). 
TABLe 1. Confidence coefficients for the significance level $\alpha=0.01$

\begin{tabular}{cccccc}
\hline Iter. No. & $\zeta_{a}$ & $\zeta_{b}$ & $\zeta_{c}$ & $P\left(\zeta_{c}\right)$ & $0.99-P\left(\zeta_{c}\right) \mid$ \\
\hline \hline 1 & 0.000000 & 0.152609 & 0.076304 & 0.515705 & 0.474295 \\
2 & 0.000000 & 0.076304 & 0.038152 & 0.762730 & 0.227270 \\
3 & 0.000000 & 0.038152 & 0.019076 & 0.882182 & 0.107818 \\
4 & 0.000000 & 0.019076 & 0.009538 & 0.940484 & 0.049516 \\
5 & 0.000000 & 0.009538 & 0.004769 & 0.970907 & 0.019093 \\
6 & 0.000000 & 0.004769 & 0.002385 & 0.985380 & 0.004620 \\
7 & 0.000000 & 0.002385 & 0.001192 & 0.992541 & 0.002541 \\
8 & 0.001192 & 0.002385 & 0.001788 & 0.988984 & 0.001016 \\
9 & 0.001192 & 0.001788 & 0.001490 & 0.990809 & 0.000809 \\
\hline
\end{tabular}

TABle 2. Confidence coefficients for the significance level $\alpha=0.025$

\begin{tabular}{cccccc}
\hline Iter. No. & $\zeta_{a}$ & $\zeta_{b}$ & $\zeta_{c}$ & $P\left(\zeta_{c}\right)$ & $0.975-P\left(\zeta_{c}\right) \mid$ \\
\hline \hline 1 & 0.000000 & 0.152609 & 0.076304 & 0.515705 & 0.459295 \\
2 & 0.000000 & 0.076304 & 0.038152 & 0.762730 & 0.212270 \\
3 & 0.000000 & 0.038152 & 0.019076 & 0.882182 & 0.092818 \\
4 & 0.000000 & 0.019076 & 0.009538 & 0.940484 & 0.034516 \\
5 & 0.000000 & 0.009538 & 0.004769 & 0.970907 & 0.004093 \\
6 & 0.000000 & 0.004769 & 0.002385 & 0.985380 & 0.010380 \\
7 & 0.002385 & 0.004769 & 0.003577 & 0.977459 & 0.002459 \\
8 & 0.003577 & 0.004769 & 0.004173 & 0.974140 & 0.000860 \\
\hline
\end{tabular}

$$
\phi(x, y)=\frac{1}{2 \pi} e^{\left(-\frac{x^{2}+y^{2}}{2}\right)}
$$

The search area is determined as $\Omega:[-4,4] \times[-4,4]$. Some probability values may be outside of the region due to the conversion of the probability density function, which is defined in an infinite space to the search area in a finite space. Therefore, the probability value in the search area is calculated as 0.998 . This probability value can be sufficient for the search area. The tolerance value $(\epsilon)$ is determined as $1 \times 10^{-3}$ and the region is also gridded according to the number of $20 \times 20$ grids. Table 1 shows the simulation results for the significance level 0.01 .

According to Table 1, the confidence coefficient 0.99 was reached in the $9^{\text {th }}$ iteration. The confidence regions obtained from each iteration are given in Figure 12(a). Figure 12(b) demonstrates the optimum confidence region for $\alpha=0.01$.

The simulation results for the significance level 0.025 are given in Table Q.

According to Table 2, the confidence coefficient 0.975 was reached in the $8^{\text {th }}$ iteration. The confidence regions obtained from each iteration are given in Figure 13 (a). Figure 13(b) demonstrates the optimum confidence region for $\alpha=0.025$. 


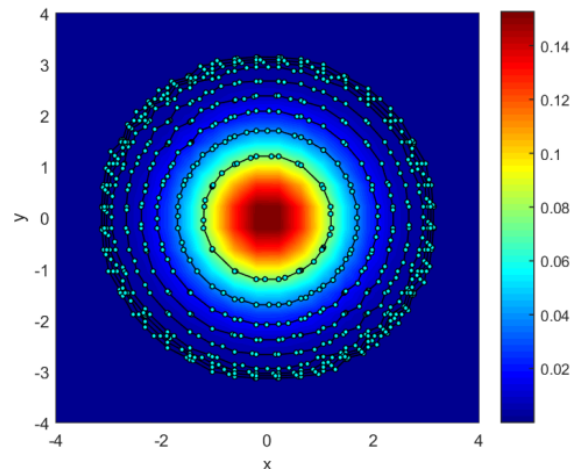

(a)

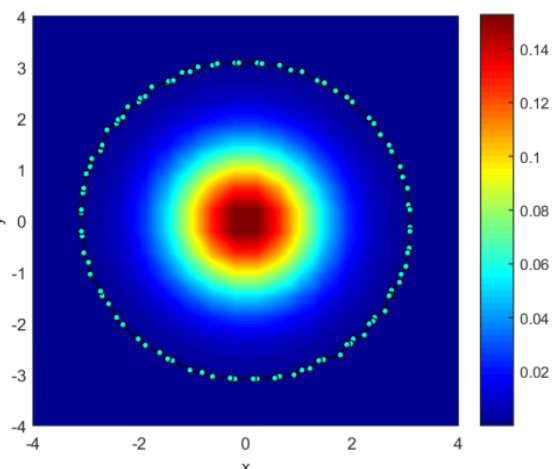

(b)

FIGURE 12. Finding the confidence region for $\alpha=0.01$, (a) The confidence regions obtained from all iterations; (b) The optimum confidence region.

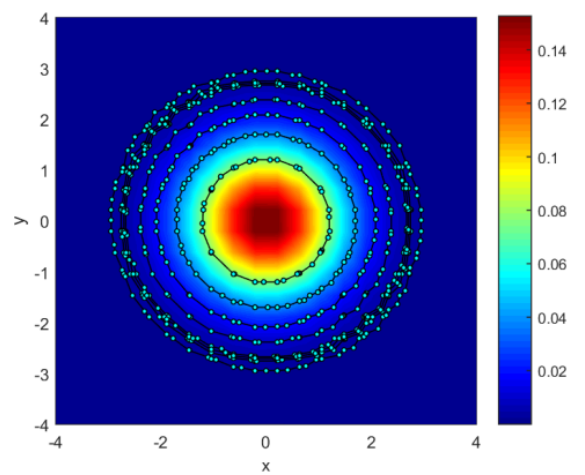

(a)

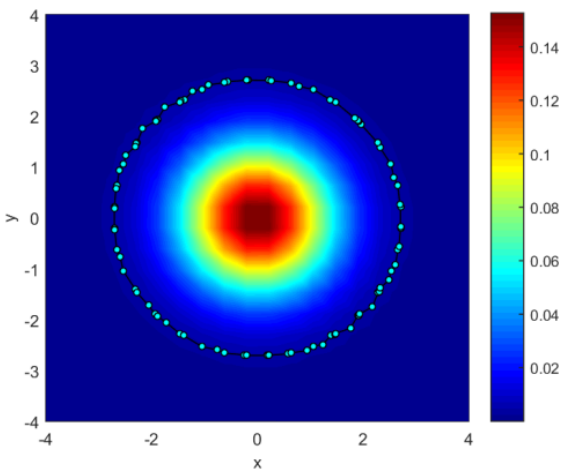

(b)

FiguRE 13. Finding the confidence region for $\alpha=0.025$, (a) The confidence regions obtained from all iterations; (b) The optimum confidence region.

Table 3 shows the simulation results for the significance level 0.05 .

According to Table 3, the confidence coefficient 0.95 was reached in the $9^{\text {th }}$ iteration. The confidence regions obtained from each iteration are given in Figure 14 (a). Figure 14(b) demonstrates the optimum confidence region for $\alpha=0.05$.

Table 4 shows the simulation results for the significance level 0.10 . 
TABle 3. Confidence coefficients for the significance level $\alpha=0.05$

\begin{tabular}{cccccc}
\hline Iter. No. & $\zeta_{a}$ & $\zeta_{b}$ & $\zeta_{c}$ & $P\left(\zeta_{c}\right)$ & $0.95-P\left(\zeta_{c}\right) \mid$ \\
\hline \hline 1 & 0.000000 & 0.152609 & 0.076304 & 0.515705 & 0.434295 \\
2 & 0.000000 & 0.076304 & 0.038152 & 0.762730 & 0.187270 \\
3 & 0.000000 & 0.038152 & 0.019076 & 0.882182 & 0.067818 \\
4 & 0.000000 & 0.019076 & 0.009538 & 0.940484 & 0.009516 \\
5 & 0.000000 & 0.009538 & 0.004769 & 0.970907 & 0.020907 \\
6 & 0.004769 & 0.009538 & 0.007154 & 0.956182 & 0.006182 \\
7 & 0.007154 & 0.009538 & 0.008346 & 0.948216 & 0.001784 \\
8 & 0.007154 & 0.008346 & 0.007750 & 0.952225 & 0.002225 \\
9 & 0.007750 & 0.008346 & 0.008048 & 0.950226 & 0.000226 \\
\hline
\end{tabular}

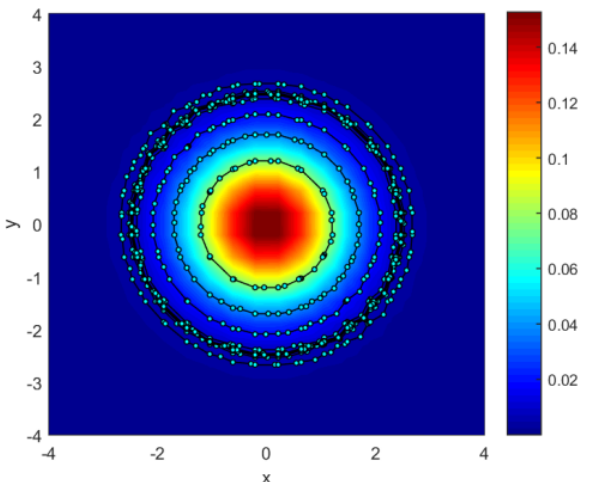

(a)

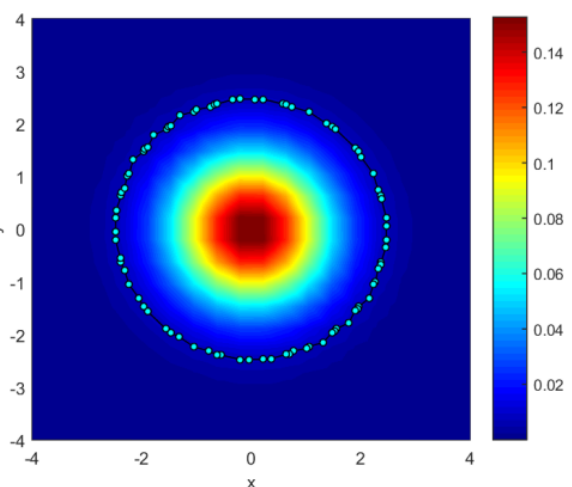

(b)

Figure 14. Finding the confidence region for $\alpha=0.05$, (a) The confidence regions obtained from all iterations; (b) The optimum confidence region.

According to Table 4, the confidence coefficient 0.90 was reached in the $9^{\text {th }}$ iteration. The confidence regions obtained from each iteration are given in Figure 15 (a). Figure 15(b) demonstrates the optimum confidence region for $\alpha=0.10$.

In order to measure the performance of the proposed method, the confidence regions for the standard normal distribution must be determined according to the given confidence coefficients. As shown in Equation (11), the desired confidence region is symmetrical in all directions. According to each of the three approaches mentioned in Section 2, the confidence region has a circular structure. Thus, the first parameter of the circle is the center point, $(0,0)$, as seen in Equation (11). To find the other parameter, the radius, the probability density function should be converted to the polar coordinate system from the Cartesian coordinate system. This 
TABLE 4. Confidence coefficients for the significance level $\alpha=0.10$

\begin{tabular}{cccccc}
\hline Iter. No. & $\zeta_{a}$ & $\zeta_{b}$ & $\zeta_{c}$ & $P\left(\zeta_{c}\right)$ & $\left|0.90-P\left(\zeta_{c}\right)\right|$ \\
\hline \hline 1 & 0.000000 & 0.152609 & 0.076304 & 0.515705 & 0.384295 \\
2 & 0.000000 & 0.076304 & 0.038152 & 0.762730 & 0.137270 \\
3 & 0.000000 & 0.038152 & 0.019076 & 0.882182 & 0.017818 \\
4 & 0.000000 & 0.019076 & 0.009538 & 0.940484 & 0.040484 \\
5 & 0.009538 & 0.019076 & 0.014307 & 0.912242 & 0.012242 \\
6 & 0.014307 & 0.019076 & 0.016692 & 0.897226 & 0.002774 \\
7 & 0.014307 & 0.016692 & 0.015499 & 0.904739 & 0.004739 \\
8 & 0.015499 & 0.016692 & 0.016095 & 0.901006 & 0.001006 \\
9 & 0.016095 & 0.016692 & 0.016394 & 0.899079 & 0.000921 \\
\hline
\end{tabular}

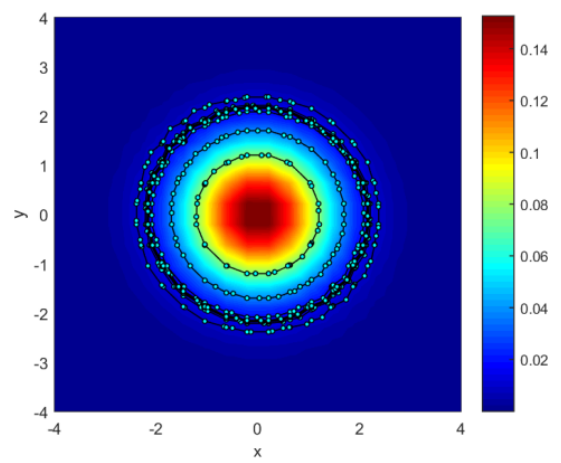

(a)

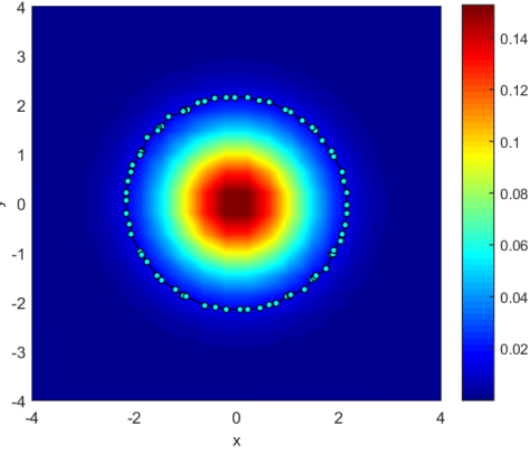

(b)

FiguRE 15. Finding the confidence region for $\alpha=0.10$, (a) The confidence regions obtained from all iterations; (b) The optimum confidence region.

function is converted as (12).

$$
\Phi(r, \theta)=\frac{1}{2 \pi} \int_{0}^{\theta} \int_{0}^{r} e^{-\frac{r^{2}}{2}} r d r d \theta
$$

Equation (12) is updated to the following equation by integrating $\theta$ into the interval $[0,2 \pi] . \theta$ is integrated due to the equality of the changes in each direction of the probability density function.

$$
\Phi(r, 2 \pi)=\Phi_{R}(r)=\int_{0}^{r} e^{-\frac{r^{2}}{2}} r d r
$$

The distribution function based on $r$ is found below.

$$
\Phi_{R}(r)=1-e^{-\frac{r^{2}}{2}}
$$


TABle 5. Radius sizes and relative absolute error

\begin{tabular}{cccc}
\hline$\alpha$ & $r_{\alpha}$ & $E_{\alpha}$ & $100\left(1-E_{\alpha}\right)$ \\
\hline \hline 0.01 & 3.0349 & 0.0197 & 98.0306 \\
0.025 & 2.7162 & 0.0168 & 98.3199 \\
0.05 & 2.4477 & 0.0144 & 98.5593 \\
0.1 & 2.1460 & 0.0162 & 98.3779 \\
\hline
\end{tabular}

The desired confidence region $\Omega$ is shown as (15).

$$
\begin{aligned}
\operatorname{Pr}((X, Y) \in \Omega) & =1-\alpha \\
& =\Phi_{R}(r)-\Phi_{R}(0)
\end{aligned}
$$

In this equation, $\Phi_{R}(0)=0$. Thus, Equation (16) is obtained by updating the equation above.

$$
\begin{aligned}
\Phi_{R}(r) & =1-\alpha \\
& =1-e^{-\frac{r^{2}}{2}}
\end{aligned}
$$

The following equation is obtained when the equation above is updated.

$$
r_{\alpha}=\sqrt{-2 \log (\alpha)}
$$

Through this equation, radius sizes with different $\alpha$ values are given Table 5 .

In order to measure the performance of the proposed method, the radius that results from Equation (17) should be compared with the confidence region determined by employing the proposed method. In order to do this, the circle and the polygon are superimposed in Figure 16(a). Also, to calculate more clearly the differences between two shapes, the points on the circle are added to the polygon (Figure 16(b)). The sectors are formed by the drawing of line segments from each polygon node to the center of the circle. Then, the relative absolute error is calculated by determining the ratio of the sum of the subtraction of the sectors and triangles with the entire area of the circle (Figure 16(c)). It is demonstrated as follows.

$$
E_{\alpha}=\frac{\sum_{i=1}^{n} \mid \text { Sector }_{i}-\text { Triangl }_{i} \mid}{\pi r_{\alpha}^{2}}
$$

The relative absolute error and percent performance rate for all significance values are shown in Table 5 .

Example 2. In this example, the aim is to determine the confidence regions for bivariate Gamma distribution within a semi-infinite space. The probability density function of the Gamma distribution is given as (19)

$$
\phi(x, y)=\frac{1}{4} x^{2} y^{2} e^{-(x+y)}
$$




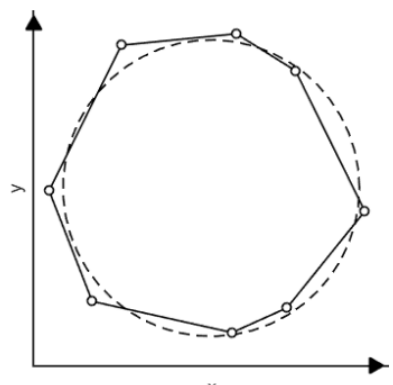

(a)

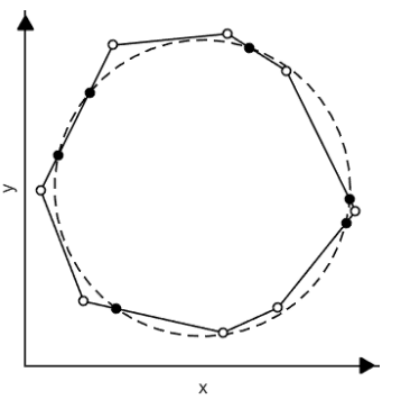

(b)

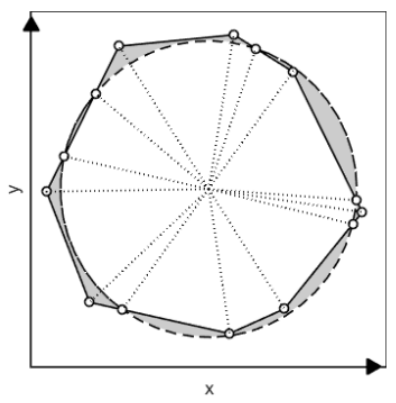

(c)

FiguRE 16. The calculation of the relative absolute error value, (a) The theoretical confidence region boundary (dashed curve) with the polygonal confidence region boundary (solid lines); (b) Determining the junction points of the boundaries (black points) and recording them to polygonal array; (c) The determination of sectors that draw lines from all points of the polygon to the center of the circle and the determination of the differences with the sector and the triangle (shaded area).

TABLE 6. Confidence coefficients for the significance level $\alpha=0.01$

\begin{tabular}{cccccc}
\hline Iter. No. & $\zeta_{a}$ & $\zeta_{b}$ & $\zeta_{c}$ & $P\left(\zeta_{c}\right)$ & $0.99-P\left(\zeta_{c}\right) \mid$ \\
\hline \hline 1 & 0.000000 & 0.072871 & 0.036436 & 0.466596 & 0.523404 \\
2 & 0.000000 & 0.036436 & 0.018218 & 0.720246 & 0.269754 \\
3 & 0.000000 & 0.018218 & 0.009109 & 0.854392 & 0.135608 \\
4 & 0.000000 & 0.009109 & 0.004554 & 0.922488 & 0.067512 \\
5 & 0.000000 & 0.004554 & 0.002277 & 0.958070 & 0.031930 \\
6 & 0.000000 & 0.002277 & 0.001139 & 0.976851 & 0.013149 \\
7 & 0.000000 & 0.001139 & 0.000569 & 0.987064 & 0.002936 \\
8 & 0.000000 & 0.000569 & 0.000285 & 0.991079 & 0.001079 \\
9 & 0.000285 & 0.000569 & 0.000427 & 0.989236 & 0.000764 \\
\hline
\end{tabular}

Table 6 shows the simulation results for the significance level 0.01 .

According to Table 6, the confidence coefficient 0.99 was reached in the $9^{\text {th }}$ iteration. The confidence regions obtained from each iteration are given in Figure $17(a)$. Figure $17(b)$ demonstrates the optimum confidence region for $\alpha=0.01$.

The simulation results for the significance level 0.025 are given in Table 7 .

According to Table 7 , the confidence coefficient 0.975 was reached in the $9^{\text {th }}$ iteration. The confidence regions obtained from each iteration are given in Figure $18($ a). Figure 18(b) demonstrates the optimum confidence region for $\alpha=0.025$. 


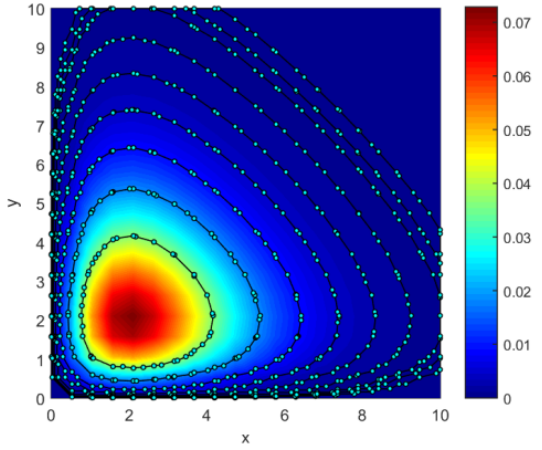

(a)

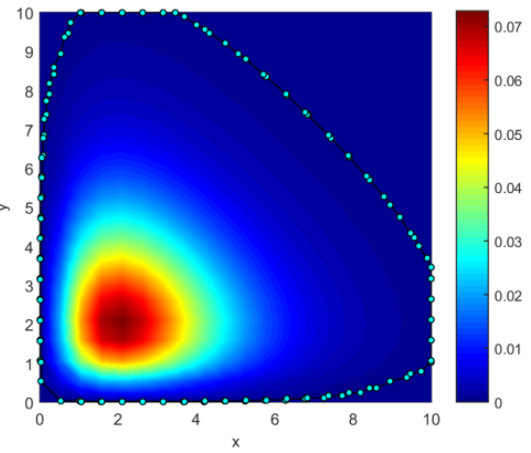

(b)

FiguRE 17. Finding the confidence region for $\alpha=0.01$, (a) The confidence regions obtained from all iterations; (b) The optimum confidence region.

TABle 7. Confidence coefficients for the significance level $\alpha=0.025$

\begin{tabular}{cccccc}
\hline Iter. No. & $\zeta_{a}$ & $\zeta_{b}$ & $\zeta_{c}$ & $P\left(\zeta_{c}\right)$ & $\left|0.975-P\left(\zeta_{c}\right)\right|$ \\
\hline \hline 1 & 0.000000 & 0.072871 & 0.036436 & 0.466596 & 0.508404 \\
2 & 0.000000 & 0.036436 & 0.018218 & 0.720246 & 0.254754 \\
3 & 0.000000 & 0.018218 & 0.009109 & 0.854392 & 0.120608 \\
4 & 0.000000 & 0.009109 & 0.004554 & 0.922488 & 0.052512 \\
5 & 0.000000 & 0.004554 & 0.002277 & 0.958070 & 0.016930 \\
6 & 0.000000 & 0.002277 & 0.001139 & 0.976851 & 0.001851 \\
7 & 0.001139 & 0.002277 & 0.001708 & 0.967380 & 0.007620 \\
8 & 0.001139 & 0.001708 & 0.001423 & 0.972095 & 0.002905 \\
9 & 0.001139 & 0.001423 & 0.001281 & 0.974489 & 0.000511 \\
\hline
\end{tabular}

Table 8 shows the simulation results for the significance level 0.05 .

According to Table 8, the confidence coefficient 0.95 was reached in the $10^{\text {th }}$ iteration. The confidence regions obtained from each iteration are given in Figure $19($ a). Figure 19(b) demonstrates the optimum confidence region for $\alpha=0.05$.

Table 9 shows the simulation results for the significance level 0.10 .

According to Table 9, the confidence coefficient 0.90 was reached in the $10^{\text {th }}$ iteration. The confidence regions obtained from each iteration are given in Figure $20($ a). Figure 20(b) demonstrates the optimum confidence region for $\alpha=0.10$.

Example 3. This example determines the confidence regions for mixture normal distribution based on the African continent as a polygonal area. Based on the pixel 


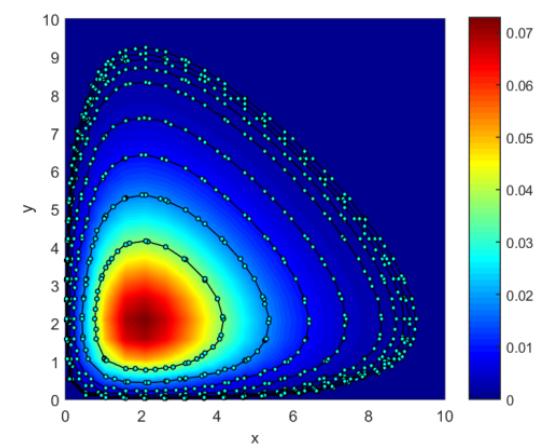

(a)

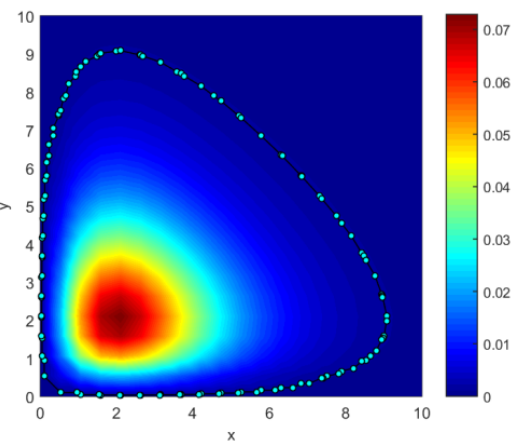

(b)

FiguRE 18. Finding the confidence region for $\alpha=0.025$, (a) The confidence regions obtained from all iterations; (b) The optimum confidence region.

TABLE 8. Confidence coefficients for the significance level $\alpha=0.05$

\begin{tabular}{cccccc}
\hline Iter. No. & $\zeta_{a}$ & $\zeta_{b}$ & $\zeta_{c}$ & $P\left(\zeta_{c}\right)$ & $0.95-P\left(\zeta_{c}\right) \mid$ \\
\hline \hline 1 & 0.000000 & 0.072871 & 0.036436 & 0.466596 & 0.483404 \\
2 & 0.000000 & 0.036436 & 0.018218 & 0.720246 & 0.229754 \\
3 & 0.000000 & 0.018218 & 0.009109 & 0.854392 & 0.095608 \\
4 & 0.000000 & 0.009109 & 0.004554 & 0.922488 & 0.027512 \\
5 & 0.000000 & 0.004554 & 0.002277 & 0.958070 & 0.008070 \\
6 & 0.002277 & 0.004554 & 0.003416 & 0.940103 & 0.009897 \\
7 & 0.002277 & 0.003416 & 0.002847 & 0.948978 & 0.001022 \\
8 & 0.002277 & 0.002847 & 0.002562 & 0.953394 & 0.003394 \\
9 & 0.002562 & 0.002847 & 0.002704 & 0.951218 & 0.001218 \\
10 & 0.002704 & 0.002847 & 0.002775 & 0.950116 & 0.000116 \\
\hline
\end{tabular}

length of the African continent, which is taken as a digital image, the probability density function is given below.

$$
\phi(x, y)=\frac{1}{3300} e^{-\frac{(x-120)^{2}+(y-60)^{2}}{1000}}+\frac{1}{4000} e^{-\frac{(x-50)^{2}+(y-125)^{2}}{700}}
$$

Table 10 shows the simulation results for the significance level 0.01 .

According to Table 10, the confidence coefficient 0.99 was reached in the $4^{\text {th }}$ iteration. The confidence regions obtained from each iteration are given in Figure 21(a). Figure 21(b) demonstrates the optimum confidence region for $\alpha=0.01$.

The simulation results for the significance level 0.025 are given in Table 11 . 


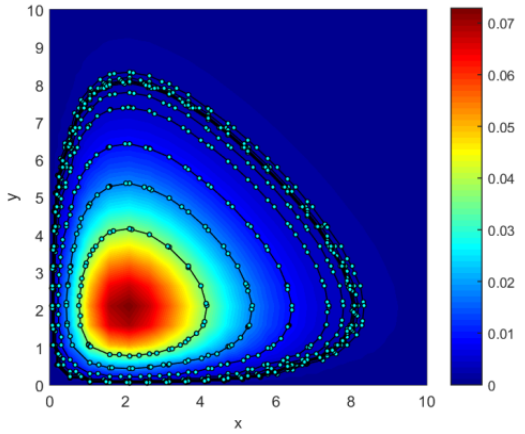

(a)

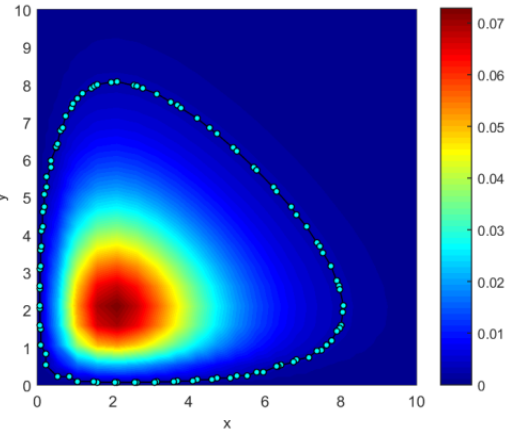

(b)

FiguRE 19. Finding the confidence region for $\alpha=0.05$, (a) The confidence regions obtained from all iterations; (b) The optimum confidence region.

TABLe 9. Confidence coefficients for the significance level $\alpha=0.10$

\begin{tabular}{cccccc}
\hline Iter. No. & $\zeta_{a}$ & $\zeta_{b}$ & $\zeta_{c}$ & $P\left(\zeta_{c}\right)$ & $0.90-P\left(\zeta_{c}\right) \mid$ \\
\hline \hline 1 & 0.000000 & 0.072871 & 0.036436 & 0.466596 & 0.433404 \\
2 & 0.000000 & 0.036436 & 0.018218 & 0.720246 & 0.179754 \\
3 & 0.000000 & 0.018218 & 0.009109 & 0.854392 & 0.045608 \\
4 & 0.000000 & 0.009109 & 0.004554 & 0.922488 & 0.022488 \\
5 & 0.004554 & 0.009109 & 0.006832 & 0.888147 & 0.011853 \\
6 & 0.004554 & 0.006832 & 0.005693 & 0.905481 & 0.005481 \\
7 & 0.005693 & 0.006832 & 0.006262 & 0.896776 & 0.003224 \\
8 & 0.005693 & 0.006262 & 0.005978 & 0.901152 & 0.001152 \\
9 & 0.005978 & 0.006262 & 0.006120 & 0.898973 & 0.001027 \\
10 & 0.005978 & 0.006120 & 0.006049 & 0.900064 & 0.000064 \\
\hline
\end{tabular}

TABle 10. Confidence coefficients for the significance level $\alpha=0.01$

\begin{tabular}{cccccc}
\hline Iter. No. & $\zeta_{a}$ & $\zeta_{b}$ & $\zeta_{c}$ & $P\left(\zeta_{c}\right)$ & $\left|0.99-P\left(\zeta_{c}\right)\right|$ \\
\hline \hline 1 & 0.000000 & 0.000301 & 0.000151 & 0.513829 & 0.476171 \\
2 & 0.000000 & 0.000151 & 0.000076 & 0.811344 & 0.178656 \\
3 & 0.000000 & 0.000076 & 0.000038 & 0.947257 & 0.042743 \\
4 & 0.000000 & 0.000038 & 0.000019 & 0.990765 & 0.000765 \\
\hline
\end{tabular}

According to Table 11, the confidence coefficient 0.975 was reached in the $5^{\text {th }}$ iteration. The confidence regions obtained from each iteration are given in Figure 22(a). Figure 22(b) demonstrates the optimum confidence region for $\alpha=0.025$. 


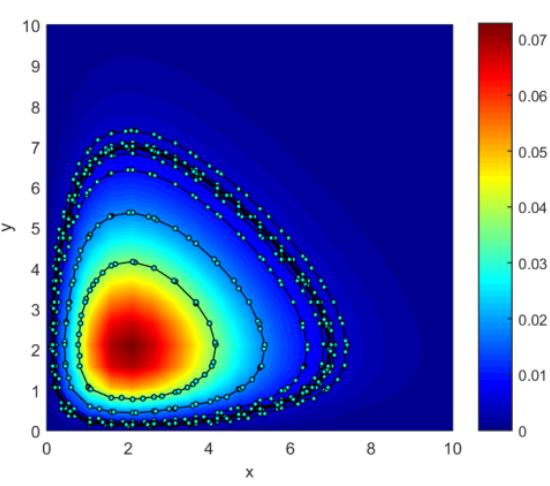

(a)

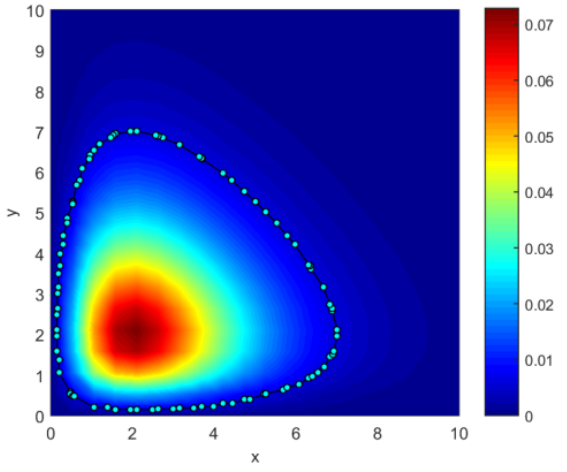

(b)

FIGURE 20. Finding the confidence region for $\alpha=0.10$, (a) The confidence regions obtained from all iterations; (b) The optimum confidence region.

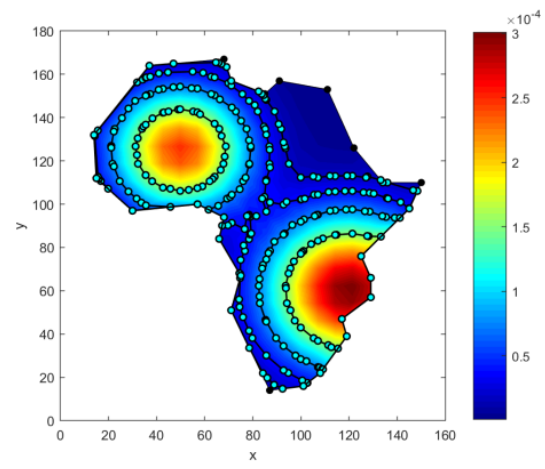

(a)

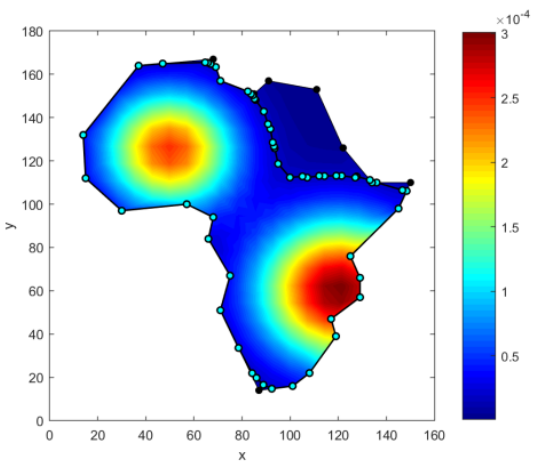

(b)

FIGURE 21. Finding the confidence region for $\alpha=0.01$, (a) The confidence regions obtained from all iterations; (b) The optimum confidence region.

Table 12 shows the simulation results for the significance level 0.05 .

According to Table 12, the confidence coefficient 0.95 was reached in the $9^{\text {th }}$ iteration. The confidence regions obtained from each iteration are given in Figure $23($ a). Figure 23(b) demonstrates the optimum confidence region for $\alpha=0.05$.

Table 13 shows the simulation results for the significance level 0.10. 
TABle 11. Confidence coefficients for the significance level $\alpha=0.025$

\begin{tabular}{cccccc}
\hline Iter. No. & $\zeta_{a}$ & $\zeta_{b}$ & $\zeta_{c}$ & $P\left(\zeta_{c}\right)$ & $0.975-P\left(\zeta_{c}\right) \mid$ \\
\hline \hline 1 & 0.000000 & 0.000301 & 0.000151 & 0.513829 & 0.461171 \\
2 & 0.000000 & 0.000151 & 0.000076 & 0.811344 & 0.163656 \\
3 & 0.000000 & 0.000076 & 0.000038 & 0.947257 & 0.027743 \\
4 & 0.000000 & 0.000038 & 0.000019 & 0.990765 & 0.015765 \\
5 & 0.000019 & 0.000038 & 0.000029 & 0.975359 & 0.000359 \\
\hline
\end{tabular}

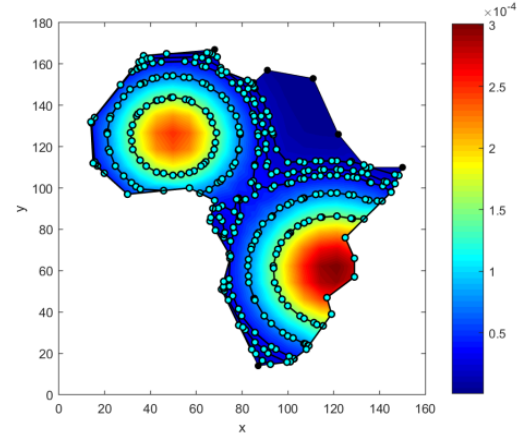

(a)

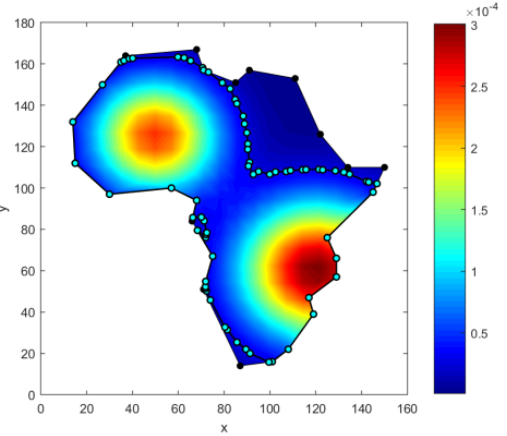

(b)

FiguRE 22. Finding the confidence region for $\alpha=0.025$, (a) The confidence regions obtained from all iterations; (b) The optimum confidence region.

TABLE 12. Confidence coefficients for the significance level $\alpha=0.05$

\begin{tabular}{cccccc}
\hline Iter. No. & $\zeta_{a}$ & $\zeta_{b}$ & $\zeta_{c}$ & $P\left(\zeta_{c}\right)$ & $0.95-P\left(\zeta_{c}\right) \mid$ \\
\hline \hline 1 & 0.000000 & 0.000301 & 0.000151 & 0.513829 & 0.436171 \\
2 & 0.000000 & 0.000151 & 0.000076 & 0.811344 & 0.138656 \\
3 & 0.000000 & 0.000076 & 0.000038 & 0.947257 & 0.002743 \\
4 & 0.000000 & 0.000038 & 0.000019 & 0.990765 & 0.040765 \\
5 & 0.000019 & 0.000038 & 0.000029 & 0.975359 & 0.025359 \\
6 & 0.000029 & 0.000038 & 0.000033 & 0.963739 & 0.013739 \\
7 & 0.000033 & 0.000038 & 0.000036 & 0.956269 & 0.006269 \\
8 & 0.000036 & 0.000038 & 0.000037 & 0.951965 & 0.001965 \\
9 & 0.000037 & 0.000038 & 0.000037 & 0.949513 & 0.000487 \\
\hline
\end{tabular}

According to Table 13, the confidence coefficient 0.90 was reached in the $9^{\text {th }}$ iteration. The confidence regions obtained from each iteration are given in Figure 24(a). Figure 24(b) demonstrates the optimum confidence region for $\alpha=0.10$. 


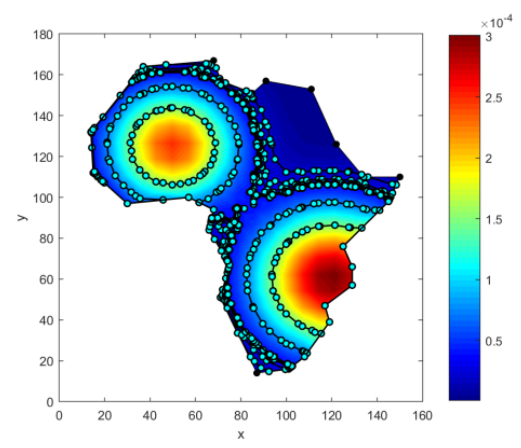

(a)

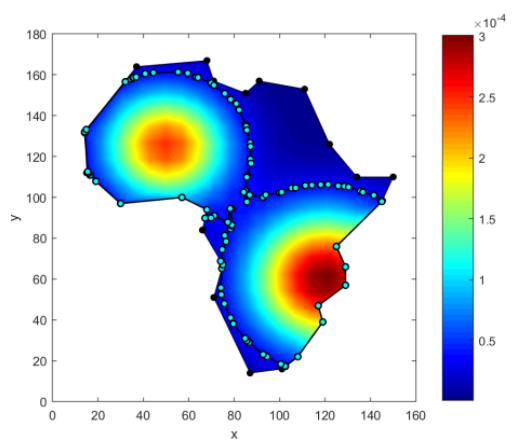

(b)

Figure 23. Finding the confidence region for $\alpha=0.05$, (a) The confidence regions obtained from all iterations; (b) The optimum confidence region.

TABle 13. Confidence coefficients for the significance level $\alpha=0.10$

\begin{tabular}{cccccc}
\hline Iter. No. & $\zeta_{a}$ & $\zeta_{b}$ & $\zeta_{c}$ & $P\left(\zeta_{c}\right)$ & $0.90-P\left(\zeta_{c}\right) \mid$ \\
\hline \hline 1 & 0.000000 & 0.000301 & 0.000151 & 0.513829 & 0.386171 \\
2 & 0.000000 & 0.000151 & 0.000076 & 0.811344 & 0.088656 \\
3 & 0.000000 & 0.000076 & 0.000038 & 0.947257 & 0.047257 \\
4 & 0.000038 & 0.000076 & 0.000057 & 0.881265 & 0.018735 \\
5 & 0.000038 & 0.000057 & 0.000047 & 0.914899 & 0.014899 \\
6 & 0.000047 & 0.000057 & 0.000052 & 0.898041 & 0.001959 \\
7 & 0.000047 & 0.000052 & 0.000050 & 0.906344 & 0.006344 \\
8 & 0.000050 & 0.000052 & 0.000051 & 0.902225 & 0.002225 \\
9 & 0.000051 & 0.000052 & 0.000051 & 0.900138 & 0.000138 \\
\hline
\end{tabular}

Example 4. In this example, earthquake risk distribution of Sivas province is modeled linearly according to North Anatolian fault line [23] and its confidence region is estimated. Based on the pixel length of the Sivas province, which is taken as a digital image, the probability density function is given below.

$$
\phi(x, y)=\frac{155-\left|(x-150) \cos \left(\frac{17 \pi}{12}\right)+(y-160) \sin \left(\frac{17 \pi}{12}\right)\right|}{1.6 \times 10^{6}}
$$

In (21), the decreasing earthquake intensity risk in the direction parallel to the fault line is modeled as a linear decreasing probability density function with respect to the fault line.

Table 14 shows the simulation results for the significance level 0.01 . 


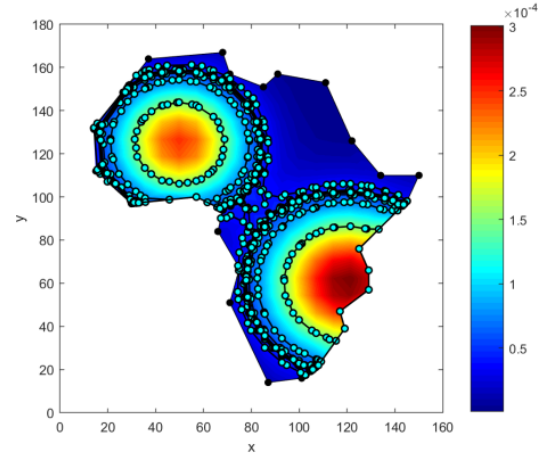

(a)

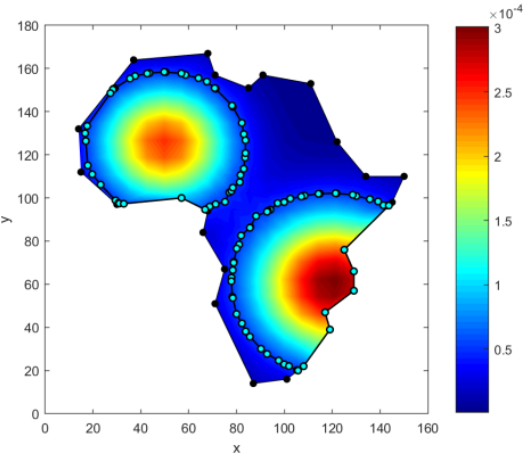

(b)

FiguRE 24. Finding the confidence region for $\alpha=0.10$, (a) The confidence regions obtained from all iterations; (b) The optimum confidence region.

TABLE 14. Confidence coefficients for the significance level $\alpha=0.01$

\begin{tabular}{cccccc}
\hline Iter. No. & $\zeta_{a}$ & $\zeta_{b}$ & $\zeta_{c}$ & $P\left(\zeta_{c}\right)$ & $0.99-P\left(\zeta_{c}\right) \mid$ \\
\hline \hline 1 & 0.000000 & 0.000097 & 0.000049 & 0.805538 & 0.184462 \\
2 & 0.000000 & 0.000049 & 0.000024 & 0.980396 & 0.009604 \\
3 & 0.000000 & 0.000024 & 0.000012 & 0.995995 & 0.005995 \\
4 & 0.000012 & 0.000024 & 0.000018 & 0.989925 & 0.000075 \\
\hline
\end{tabular}

TABLE 15. Confidence coefficients for the significance level $\alpha=0.025$

\begin{tabular}{cccccc}
\hline Iter. No. & $\zeta_{a}$ & $\zeta_{b}$ & $\zeta_{c}$ & $P\left(\zeta_{c}\right)$ & $\left|0.975-P\left(\zeta_{c}\right)\right|$ \\
\hline \hline 1 & 0.000000 & 0.000097 & 0.000049 & 0.805538 & 0.169462 \\
2 & 0.000000 & 0.000049 & 0.000024 & 0.980396 & 0.005396 \\
3 & 0.000024 & 0.000049 & 0.000037 & 0.919918 & 0.055082 \\
4 & 0.000024 & 0.000037 & 0.000031 & 0.958831 & 0.016169 \\
5 & 0.000024 & 0.000031 & 0.000028 & 0.971512 & 0.003488 \\
6 & 0.000024 & 0.000028 & 0.000026 & 0.976528 & 0.001528 \\
7 & 0.000026 & 0.000028 & 0.000027 & 0.974098 & 0.000902 \\
\hline
\end{tabular}

According to Table 14, the confidence coefficient 0.99 was reached in the $4^{\text {th }}$ iteration. The confidence regions obtained from each iteration are given in Figure 25 (a). Figure 25(b) demonstrates the optimum confidence region for $\alpha=0.01$.

The simulation results for the significance level 0.025 are given in Table 15. 


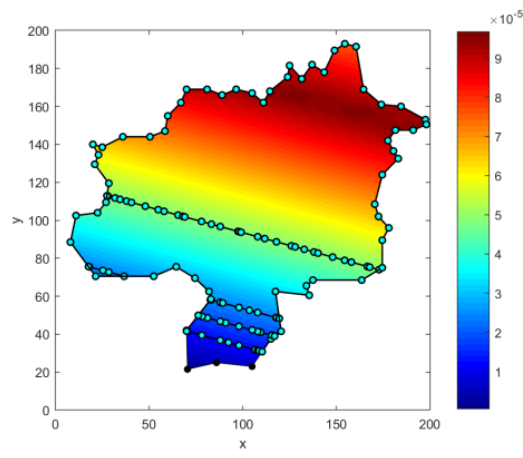

(a)

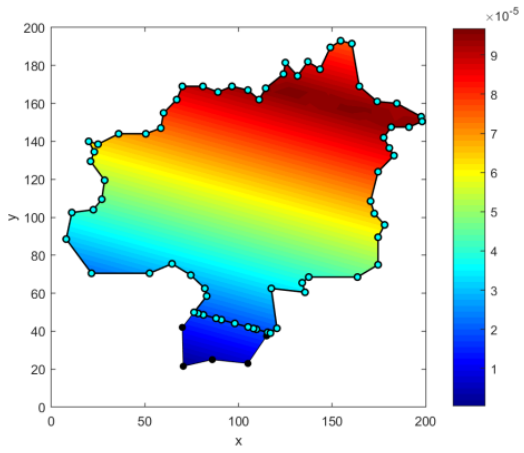

(b)

FIGURE 25. Finding the confidence region for $\alpha=0.01$, (a) The confidence regions obtained from all iterations; (b) The optimum confidence region.

According to Table 15, the confidence coefficient 0.975 was reached in the $7^{\text {th }}$ iteration. The confidence regions obtained from each iteration are given in Figure 26(a). Figure 26(b) demonstrates the optimum confidence region for $\alpha=0.025$.

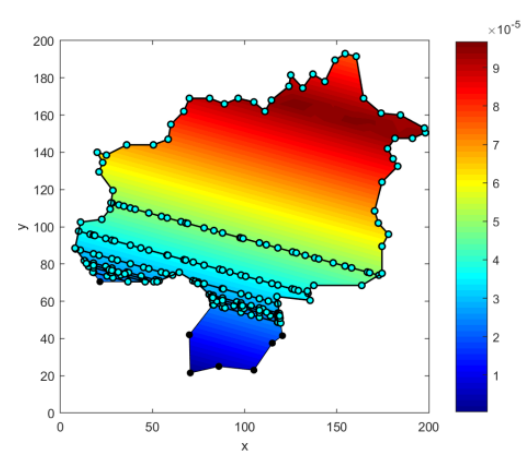

(a)

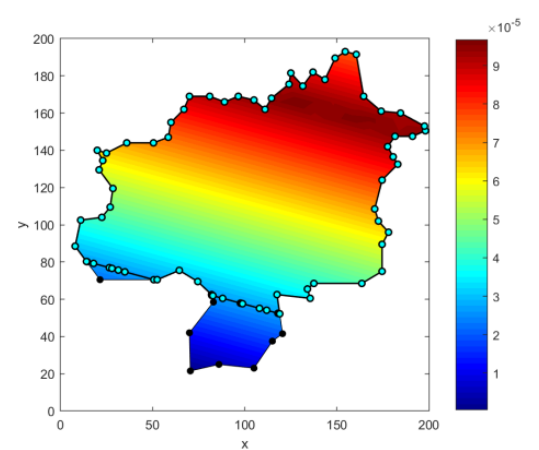

(b)

FIGURE 26. Finding the confidence region for $\alpha=0.025$, (a) The confidence regions obtained from all iterations; (b) The optimum confidence region.

Table 16 shows the simulation results for the significance level 0.05 .

According to Table 16, the confidence coefficient 0.95 was reached in the $6^{\text {th }}$ iteration. The confidence regions obtained from each iteration are given in Figure 27(a). Figure 27(b) demonstrates the optimum confidence region for $\alpha=0.05$. 
TABLE 16. Confidence coefficients for the significance level $\alpha=0.05$

\begin{tabular}{cccccc}
\hline Iter. No. & $\zeta_{a}$ & $\zeta_{b}$ & $\zeta_{c}$ & $P\left(\zeta_{c}\right)$ & $0.95-P\left(\zeta_{c}\right) \mid$ \\
\hline \hline 1 & 0.000000 & 0.000097 & 0.000049 & 0.805538 & 0.144462 \\
2 & 0.000000 & 0.000049 & 0.000024 & 0.980396 & 0.030396 \\
3 & 0.000024 & 0.000049 & 0.000037 & 0.919918 & 0.030082 \\
4 & 0.000024 & 0.000037 & 0.000031 & 0.958831 & 0.008831 \\
5 & 0.000031 & 0.000037 & 0.000034 & 0.941625 & 0.008375 \\
6 & 0.000031 & 0.000034 & 0.000032 & 0.950717 & 0.000717 \\
\hline
\end{tabular}

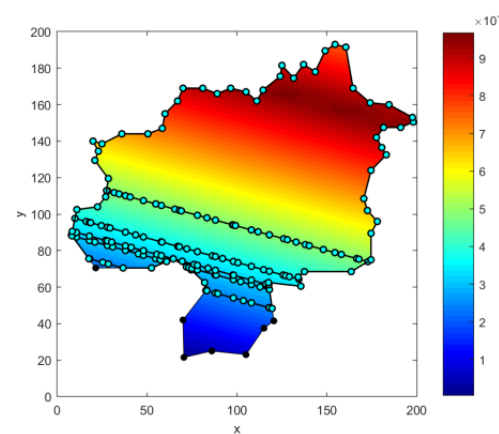

(a)

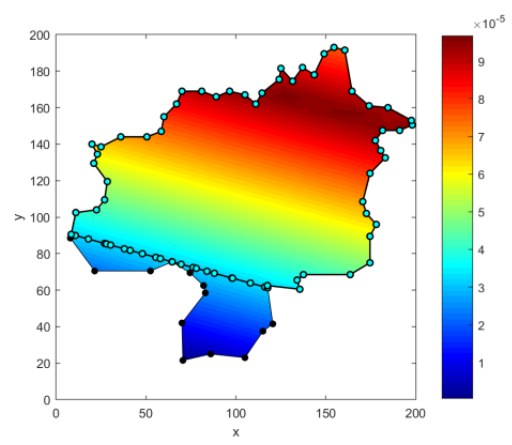

(b)

FiguRE 27. Finding the confidence region for $\alpha=0.05$, (a) The confidence regions obtained from all iterations; (b) The optimum confidence region.

TABLE 17. Confidence coefficients for the significance level $\alpha=0.10$

\begin{tabular}{cccccc}
\hline Iter. No. & $\zeta_{a}$ & $\zeta_{b}$ & $\zeta_{c}$ & $P\left(\zeta_{c}\right)$ & $0.90-P\left(\zeta_{c}\right) \mid$ \\
\hline \hline 1 & 0.000000 & 0.000097 & 0.000049 & 0.805538 & 0.094462 \\
2 & 0.000000 & 0.000049 & 0.000024 & 0.980396 & 0.080396 \\
3 & 0.000024 & 0.000049 & 0.000037 & 0.919918 & 0.019918 \\
4 & 0.000037 & 0.000049 & 0.000043 & 0.869624 & 0.030376 \\
5 & 0.000037 & 0.000043 & 0.000040 & 0.896214 & 0.003786 \\
6 & 0.000037 & 0.000040 & 0.000038 & 0.908369 & 0.008369 \\
7 & 0.000038 & 0.000040 & 0.000039 & 0.902369 & 0.002369 \\
8 & 0.000039 & 0.000040 & 0.000039 & 0.899311 & 0.000689 \\
\hline
\end{tabular}

Table 17 shows the simulation results for the significance level 0.10 . 
According to Table 17, the confidence coefficient 0.90 was reached in the $8^{\text {th }}$ iteration. The confidence regions obtained from each iteration are given in Figure 28(a). Figure 28(b) demonstrates the optimum confidence region for $\alpha=0.10$.

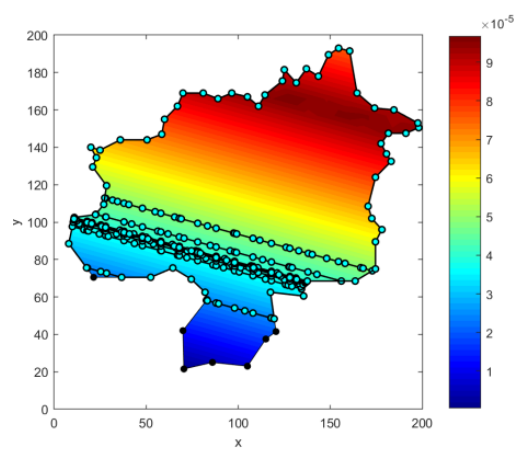

(a)

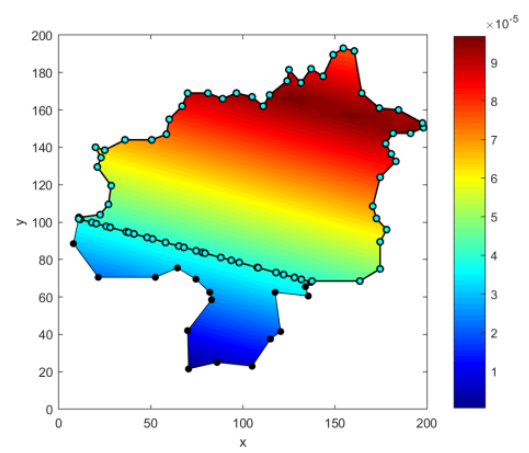

(b)

FiguRE 28. Finding the confidence region for $\alpha=0.10$, (a) The confidence regions obtained from all iterations; (b) The optimum confidence region.

\section{CONCLUSions}

This study proposes a practical method to in the attempt to modify the univariate probability density functions into the bivariate probability density functions. In the proposed method, the polygonal area is firstly separated into grids and then triangulation is applied. If the number of grids increases the performance of method can increase as well. According to the examples, the performance of the proposed method is considerably high. In addition, the proposed method provides a solution for infinite, semi-infinite and polygonal restricted areas. According to this method, the confidence region is found no matter how complex the distribution function. Confidence regions cannot be as precisely calculated in many applications. Therefore, the proposed method gives more efficient results for multimodal probability density functions.

\section{REFERENCES}

[1] Neyman, J., Outline of a theory of statistical estimation based on the classical theory of probability, Philosophical Transactions of the Royal Society of London. Series A, Mathematical and Physical Sciences 236(767), (1937), 333-380.

[2] Tate, R. F., and Klett, G. W., Optimal confidence intervals for the variance of a normal distribution, Journal of the American statistical Association 54(287), (1959), 674-682.

[3] Dunn, O., Multiple comparisons among means, Journal of the American Statistical Association 56(293), (1961), 52-64.

[4] Dunn, O., Multiple comparisons using rank sums, Technometrics 6(3), (1964), 241-252. 
[5] Chew, V., Confidence, prediction, and tolerance regions for the multivariate normal distribution, Journal of the American Statistical Association 61(315), (1966), 605-617.

[6] Sidak, Z., Rectangular confidence regions for the means of multivariate normal distributions, Journal of the American Statistical Association 62(318), (1967), 626-633.

[7] Hu, Z., and Yang, R.-C., A new distribution-free approach to constructing the confidence region for multiple parameters, PloS one 8(12), (2013), e81179.

[8] Mammen, E., and Polonik, W., Confidence regions for level sets, Journal of Multivariate Analysis 122, (2013), 202-214.

[9] Martin, R., Random sets and exact confidence regions, Sankhya A 76(2), (2014), 288-304.

[10] Rambaud-Althaus, C., Althaus, F., Genton, B., and D'Acremont, V., Clinical features for diagnosis of pneumonia in children younger than 5 years: a systematic review and metaanalysis, The Lancet Infectious Diseases 15(4), (2015), 439-450.

[11] Harrar, S., and Xu, J., Confidence regions for level differences in growth curve models, Journal of Statistical Planning and Inference 175, (2016), 11-24.

[12] Öztürk, F., and Karabulut, İ.,Interval Estimators for the Parameters of the Normal Distribution, Commun.Fac.Sci.Univ.Ank.Series A1 55(1), (2006), 23-32.

[13] Ünsal, Ü., Determining Polygonal Confidence Zone with Artificial Bee Colony Algorithm and an Application in Mines, MSc Thesis, Karadeniz Technical University, Turkey, 2014.

[14] Kesemen O., Tiryaki, B. K., Özkul, E. and Tezel, Ö., Determination of the Confidence Intervals for Multimodal Probability Density Functions, Gazi University Journal of Science 31(1), (2018) 310-326.

[15] Kesemen O., Tiryaki, B. K. and Uluyurt, T., A Novel Approximation for Computation Bivariate Distribution Functions in Polygonal Area, Gümüşhane Üniversitesi Fen Bilimleri Enstitüsü Dergisi 9(1), (2019) 88-98.

[16] Douglas, D., and Peucker, T., Algorithms for the reduction of the number of points required to represent a digitized line or its caricature, Cartographica: The International Journal for Geographic Information and Geovisualization 10(2), (1973), 112-122.

[17] Kesemen O. and Tiryaki, B. K., Non-Uniform Random Number Generation from Arbitrary Bivariate Distribution in Polygonal Area, Süleyman Demirel Üniversitesi Fen Bilimleri Enstitüsü Dergisi 22(2), (2018), 443-457.

[18] Chew, L., Constrained delaunay triangulations, Algorithmica 4(1-4), (1989), 97-108.

[19] Thacker, W. C., A brief review of techniques for generating irregular computational grids, International Journal for Numerical Methods in Engineering 15(9), (1980), 1335-1341.

[20] Fulton, S. R., Ciesielski, P. E., and Schubert, W. H., Multigrid methods for elliptic problems: A review. Monthly Weather Review 114 (5), (1986), 943-959.

[21] Sulman, M., Williams, J. F., and Russell, R. D., Optimal mass transport for higher dimensional adaptive grid generation, Journal of computational physics 230(9), (2011), 3302-3330.

[22] Stilitz, I., and Yitzhaky, J., The effect of grid size on street location time in maps, Applied ergonomics 10(4), (1979), 235-239.

[23] Özmen, B., Nurlu, M., and Güler, H., Coğrafi Bilgi Sistemi ile Deprem Bölgelerinin İncelenmesi, Bayındırlık ve İskan Bakanlığı, Afet İşleri Genel Müdürlüğü, Ankara, 1997. 
Current address: ORHAN KESEMEN: Department of Statistics and Computer Sciences, Karadeniz Technical University, 61080 Trabzon, Turkey.

E-mail address: okesemen@gmail.com

ORCID Address: http://orcid.org/0000-0002-5160-1178

Current address: Eda ÖZKUL: Department of Statistics and Computer Sciences, Karadeniz Technical University, 61080 Trabzon, Turkey.

E-mail address: eda.ozkul.gs@gmail.com

ORCID Address: http://orcid.org/0000-0002-9840-8818

Current address: Ülkü ÜNSAL: Department of of Health Management, Karadeniz Technical University, 61080 Trabzon, Turkey.

E-mail address: ulkunsal@gmail.com

ORCID Address: http://orcid.org/0000-0002-8363-1806 